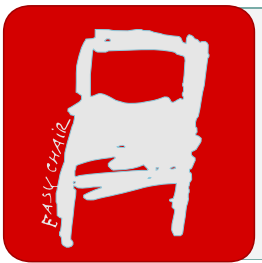

EPiC Series in Health Sciences

Volume 1, 2017, Pages 296-303

CAOS 2017. 17th Annual Meeting of the International

Society for Computer Assisted Orthopaedic Surgery

\title{
Computer navigated reduction of scaphoid non-unions and displaced scaphoid fractures- a cadaveric study
}

\author{
Michael Chu-Kay Mak ${ }^{1}$, Elvis Chun-Sing Chui ${ }^{2}$, Wing-Lim Tse ${ }^{3}$, Pak-Cheong Ho ${ }^{4}$ \\ ${ }^{1}$ Department of Orthopaedics and Traumatology, Prince of Wales Hospital, The \\ Chinese University of Hong Kong, Hong Kong
}

Scaphoid non-union results in multiplanar deformities between the fracture fragments. These give rise to the typical humpback deformity, pronation and ulnar deviation of the distal fragment, and a bone defect in the non-union site with shortening. Bone grafting, whether open or arthroscopic, relies on fluoroscopic and direct visual assessment of reduction. However, because of the bone defect and irregular geometry, it is difficult to determine the precise width of the bone gap and restore the original bone length, and to correct interfragmentary rotation. Malunion is a typical complication of surgery for scaphoid non-union, with satisfactory outcomes in only $27 \%$ if there is a significant humpback deformity (1). The use of intraoperative 3D imaging to assess quality of reduction of scaphoid fractures and non-unions was reported (2). However, the actual correction was still performed with fluoroscopy. Schweizer et al. reported the use of computer-based planning and the use of patient-specific guides in the reduction of scaphoid fragments in non-unions and fractures (3). An open approach with significant dissection and exposure of the scaphoid was required to fit the guide onto the bone fragments, which may disrupt the tenuous blood supply. Other authors have reported the use of computer navigation in guiding screw insertion in non-displaced scaphoid fractures (4). In these techniques, the wrist was immobilized in a splint which incorporated the tracker device. Screw insertion was navigated to improve accuracy and decrease radiation dose. However, the use of computer navigation in guiding reduction in scaphoid non-unions and displaced fractures have not been reported. 
Computer navigated reduction of scaphoid ... M. C.-K. Mak, E. C.-S. Chui, W.-L. Tse and P.-C. Ho

Objective

We propose a method of anatomical reconstruction in scaphoid non-union by computer-assisted preoperative planning combined with intraoperative computer navigation. This could be done in conjunction with a minimally invasive, arthroscopic bone grafting technique, which was pioneered in our center by the senior author (Ho, P.C.). In this way, accurate correction of deformity could be achieved while preserving vascularity and limiting soft tissue trauma to the wrist.

Methods:

Two models were used to establish the workflow and feasibility of this method respectively. Using cadaveric wrists, contralateral scaphoids were not available for mirroring. For the purposes of this pilot study we used computed tomography (CT) images of the intact/ reconstituted scaphoid for reference instead. Fixation was achieved by multiple K-wires as this is the preferred method in the 20 years of experience of bone grafting of the scaphoid in our center. For the purpose of deformity correction in this study, $2 \mathrm{~K}$-wires were navigated to achieve correction of rotation.

The first model consisted of a cadaveric scaphoid bone and a patient tracker. The scaphoid bone was harvested from a cadaver with no prior wrist trauma and stripped of soft tissue. A CT scan (GE VCT 64) of the scaphoid with $0.625 \mathrm{~mm}$ slice thickness was obtained. A fracture was then created with an osteotome in the scaphoid waist. The proximal fragment was pinned to a model of the radius bone at the scaphoid fossa with a $1.6 \mathrm{~mm}$ titanium K-wire. Simulating a fixation procedure from the volar approach, 2 titanium K-wires of $1.25 \mathrm{~mm}$ diameter were driven into the distal fragment, and tagged to the proximal pole in a displaced position (Fig. 1). Together with the patient tracker that was fixed onto its base, the model was scanned with a 3D C-arm (Arcadis Orbic 3D, Siemens AG, Erlangen, Germany). The proximal and distal fragments in the acquired images were matched and fused with the CT images of the intact scaphoid using a 3D image processing software (Mimics 18.0, Materialise, Leuven, Belgium). The initial image with the displaced fragments was thus transformed into a fused image in which the fragments were in their anatomical positions. The K-wire tracts in the distal fragment were extended into the proximal fragment as planned pin tracts. This transformed image is exported into the computed navigation program (eNlite Navigation System, Stryker, Kalamazoo, USA). The K-wire tracts in the proximal fragment were recognized and planned as pin tracts. Registration in the navigation system was done by using a tracker-mounted driver holding another identical K-wire at a known length. The K-wires, held at the same length, were driven into the proximal 
fragment according to the planned pin tracts on the navigation system. This would fix the scaphoid in a reduced position. Reduction was assessed by measuring the amount of articular stepping and displacement using a caliper.

In the second model, a pair of cadaver wrists were used, which included the mid-forearm to the hand. Scanning and navigation devices were the same as those in the first model. A scaphoid waist fracture was created with an osteotome via a dorsal approach, deliberately causing a small amount of bone loss to simulate the non-union condition. A CT scan of the simulated non-union was obtained and underwent segmentation to isolate the scaphoid using the Mimics software. As with the first model, the proximal pole was fixed to the distal radius by a $1.6 \mathrm{~mm}$ titanium K-wire inserted dorsally under fluoroscopy, and $21.25 \mathrm{~mm}$ titanium $\mathrm{K}$-wires were inserted into the distal fragment, aiming for a relatively volar part so as to leave space for bone graft inserted from a dorsal to volar direction. An anchoring pin was inserted into the radial shaft at the radial aspect just dorsal to the brachioradialis muscle, and a patient tracker was mounted on it. With the K-wires and tracker in place (Fig. 2), the wrists were scanned with a 3D C-arm. The acquired images underwent manually guided segmentation using Mimics software to isolate the proximal and distal scaphoid fragments. These were matched and fused to the corresponding fragments from the CT images (Fig. 3). Reduction of the fragments was performed semi-automatically in the program, mainly by automatic point matching and fine manual adjustment and aligning the volar and dorsal edges of the midcarpal articular surface. The transformed image, with a corrected alignment and extended pin tracts in the proximal fragment, was exported to the navigation program and the extended pin tracts were again planned as targeted pin tracts. With navigation guidance, the K-wires were driven into the proximal fragment, using the same method established in model 1. Direct visual assessment of reduction was done, and due to limited visualization of the whole scaphoid in the wrist joint, a CT scan was done to assess reduction and the images were analyzed in the Mimics program.

\section{Results}

In model 1, the workflow for computer-assisted anatomical reduction of a simulated displaced scaphoid non-union was established. Navigated insertion of $\mathrm{K}$-wires resulted in a good reduction, with less than $0.5 \mathrm{~mm}$ of articular stepping, displacement, and discrepancy in length.

In model 2, the intact soft tissue envelope adversely affects resolution of the images acquired by the 3D C-arm in the second model. Manual segmentation was necessary to accurately isolate the scaphoid fragments, and manual adjustments were made to achieve matching with the CT images. The reduction 
Computer navigated reduction of scaphoid ... M. C.-K. Mak, E. C.-S. Chui, W.-L. Tse and P.-C. Ho

after navigated fixation resulted in less than $1 \mathrm{~mm}$ articular stepping, displacement, and discrepancy in length (Fig. 4).

Discussion

This study demonstrated that an accurate reduction of the scaphoid in nonunions and displaced fractures can be accurately performed using computed navigation and computer-assisted planning. It is the first report on the use of computer navigation in correction of alignment in the wrist. Computer-assisted deformity correction by placing a patient-specific guide on the scaphoid (3) or the use of a computer-generated hard model as a visual guide have been reported previously (5). However, both require open surgery which may affect vascularity of the scaphoid, and in the second technique, the reduction itself is not guided. To apply it in the in vivo non-union situation, however, there are issues that need to be solved. The segmentation of the 3D images and planning of pin trajectories need to be performed intraoperatively. This requires the availability of a team member who is familiar with image processing software and the anatomy of the scaphoid so that this can be done at the same time as the surgery. As this may take time to perform, the insertion of patient tracker and K-wires should be done at the beginning of surgery followed by 3D image acquisition. Computer planning proceeds in parallel with arthroscopic evaluation, synovectomy, debridement of the non-union site and bone graft harvesting. This part of the surgery usually requires 60 minutes. The time taken for image processing can be shortened if CT images of the contralateral scaphoid are mirrored, and those of the ipsilateral scaphoid are reduced into anatomical alignment preoperatively.

Conclusion

Computer-assisted correction of alignment of scaphoid non-union is feasible. However, it requires simultaneous computer planning during surgery, and a learning curve to improve accuracy and efficiency.

\section{Reference:}

1. Amadio PC, Berquist TH, Smith DK, Ilstrup DM, Cooney WP 3rd, Linscheid RL. Scaphoid malunion. J Hand Surg Am. 1989 Jul;14(4):67987

2. Luria S, Safran O, Zinger G, Mosheiff R, Liebergall M. Intraoperative 3-dimensional imaging of scaphoid fracture reduction and fixation. Orthop Traumatol Surg Res. 2015 May;101(3):353-7

3. Schweizer A, Mauler F, Vlachopoulos L, Nagy L, Fürnstahl P. ComputerAssisted 3-Dimensional Reconstructions of Scaphoid Fractures and 
Nonunions With and Without the Use of Patient-Specific Guides: Early Clinical Outcomes and Postoperative Assessments of Reconstruction Accuracy. J Hand Surg Am. 2016 Jan;41(1):59-69

4. Kam CC, Greenberg JA. Computer-assisted navigation for dorsal percutaneous scaphoid screw placement: a cadaveric study. J Hand Surg Am. 2014 Apr;39(4):613-20

5. Murase T, Moritomo H, Goto A, Sugamoto K, Yoshikawa H. Does threedimensional computer simulation improve results of scaphoid nonunion surgery? Clin Orthop Relat Res. 2005 May;(434):143-50

Figures:

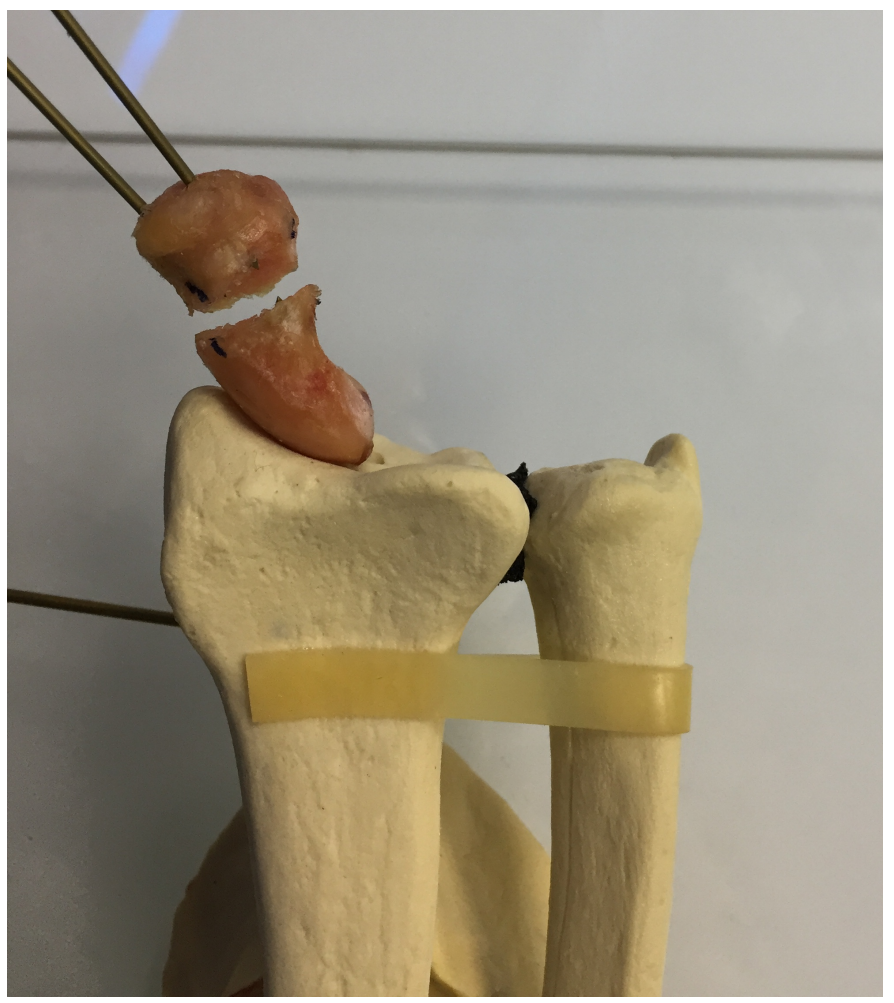

Fig. 1. The proximal fragment was fixed to the forearm model with a $1.6 \mathrm{~mm} \mathrm{~K}$ wire, and $21.25 \mathrm{~mm} \mathrm{~K}$-wires were inserted into the distal fragment. 


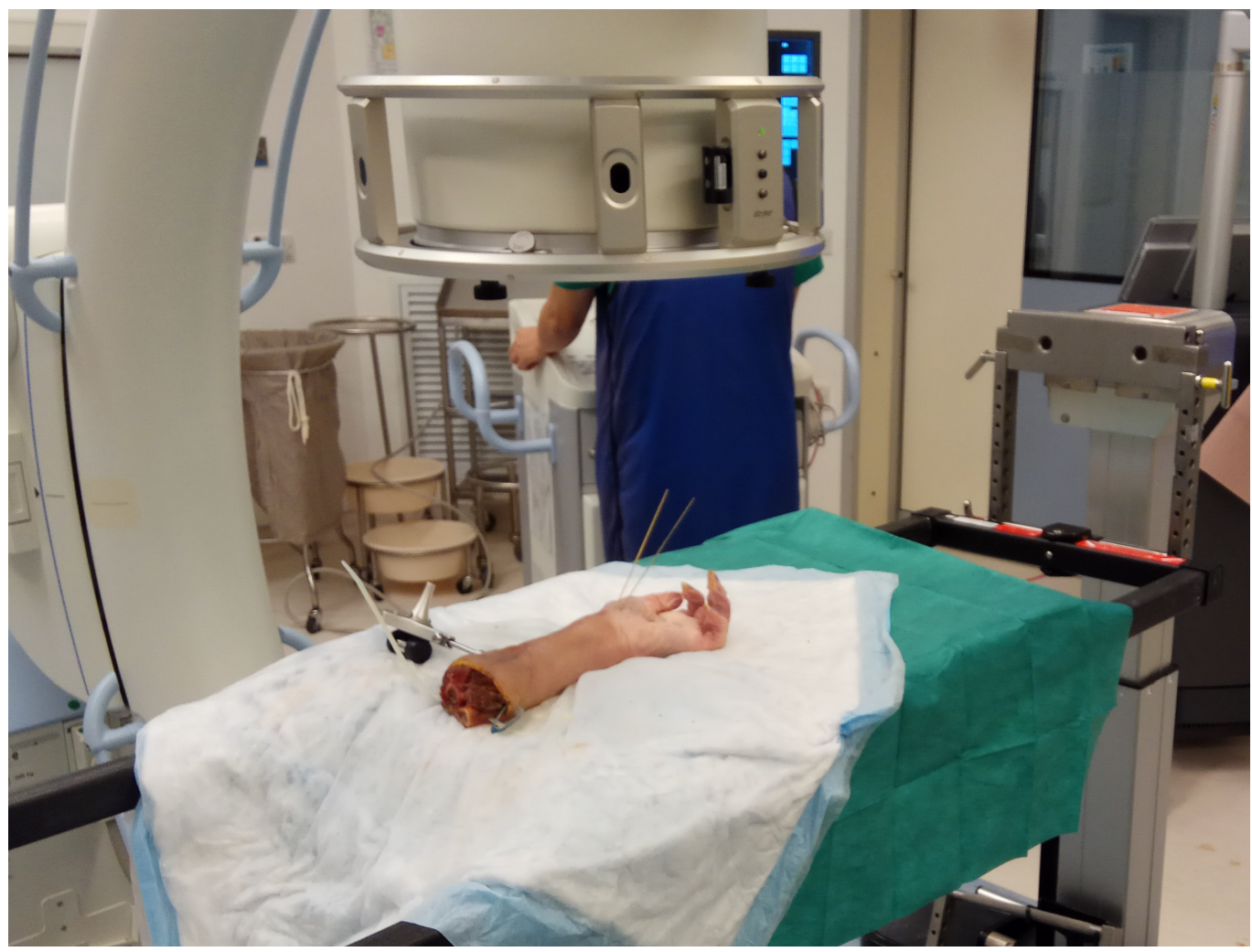

Fig 2. A patient tracker was inserted onto the radial shaft of the cadaver. The proximal fragment was fixed to the radius with a K-wire. $2 \mathrm{~K}$-wires were inserted into the distal fragment. 3D images of the wrist were then acquired. 
Computer navigated reduction of scaphoid ... M. C.-K. Mak, E. C.-S. Chui, W.-L. Tse and P.-C. Ho

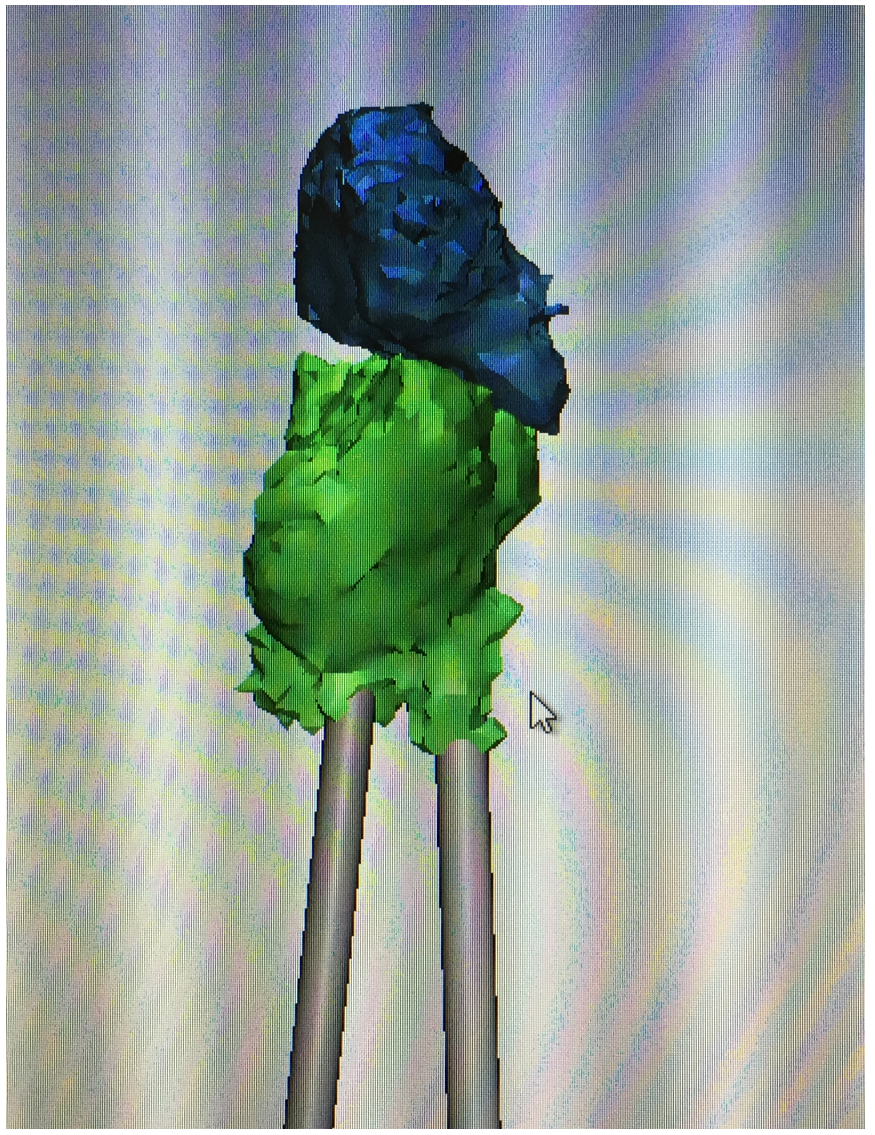

Fig. 3. The segmented 3D image, before fusion to the CT images. 
Computer navigated reduction of scaphoid ... $\quad$ M. C.-K. Mak, E. C.-S. Chui, W.-L. Tse and P.-C. Ho

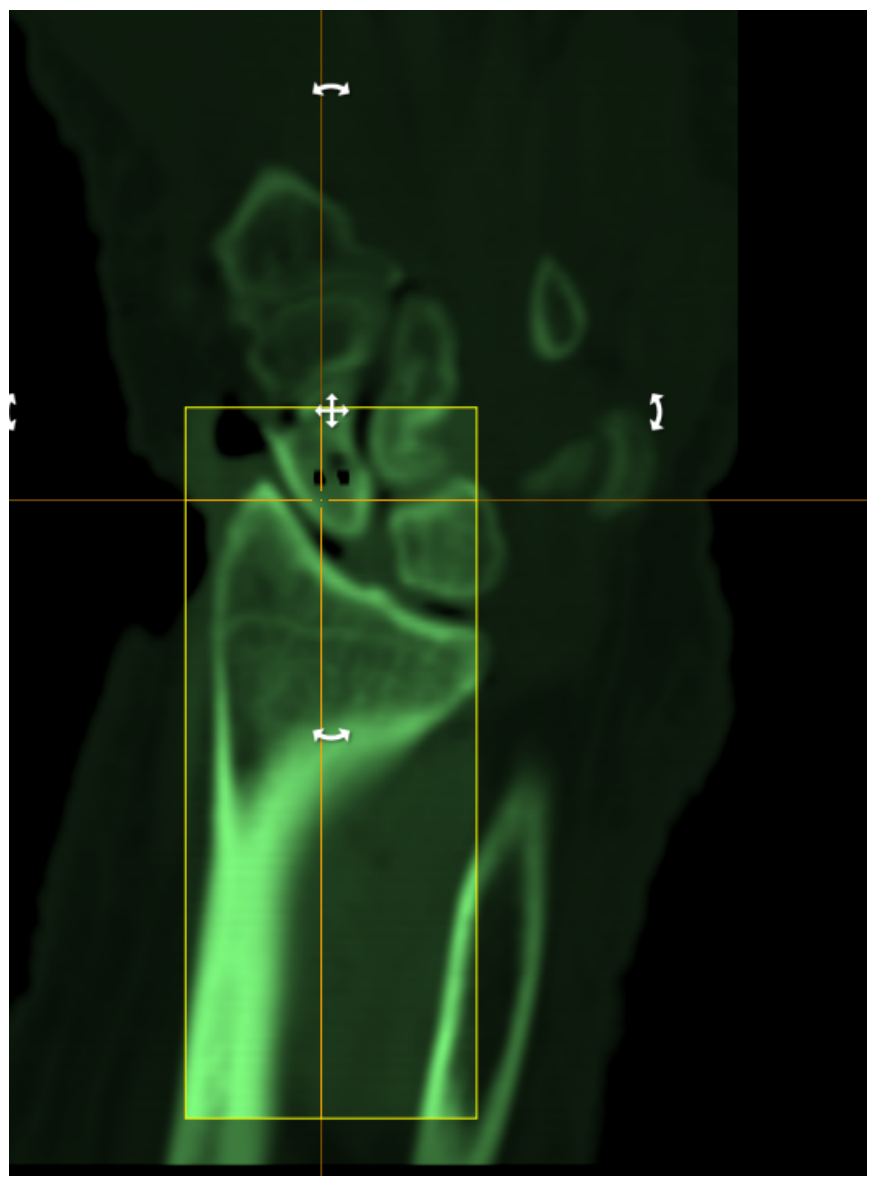

Fig. 4. Post-fixation CT image showing the scaphoid in the coronal plane in a reduced position with minimal articular stepping. 